\title{
Evolution of $\mathrm{MoO}_{3}$ nanobelts and nanoplatelets formation with flame synthesis
}

\author{
Chao Ma ${ }^{\text {a }}$, Xinyao Zou ${ }^{a}$, Ang $\mathrm{Li}^{\text {a }}$, Haozhen Li ${ }^{\text {a }}$, Stelios Rigopoulos ${ }^{\mathrm{b}}$, Lei Zhu ${ }^{\text {a, }}$, Zhen Huang ${ }^{\mathrm{a}}$ \\ ${ }^{a}$ Key Lab. for Power machinery and Engineering of M. O. E., Shanghai Jiao Tong University, Shanghai, P.R. China \\ ${ }^{\mathrm{b}}$ Department of Mechanical Engineering, Imperial College London, Exhibition Road, London SW7 2AZ, UK
}

\section{Corresponding author: Lei Zhu}

Mailing address: School of Mechanical Engineering, Shanghai Jiao Tong University, Shanghai, P.R. China.

E-mail address: tonyzhulei@gmail.com; tonyzhulei@sjtu.edu.cn (Lei Zhu*)

\section{Colloquium: SOOT, NANOMATERIALS, AND LARGE MOLECULES}

\section{Total length of paper:}

Total: $\mathbf{5 9 5 0 . 7 8}$ words

\section{- Abstract: total 179 words}

- Main text: 3783 words (MS word processor utility)

- Equations: total 0 words

$=(\#$ equation lines $+\#$ blank lines $) \times(7.6$ words/line $) \times(\#$ columns $)$.

- References: total 349.6 words

$=(\# 18+2) \times(2.3$ lines/reference $) \times(7.6$ words/line $)=349.6$ words

- Tables: total 0 words

$=(\#$ text lines +2 lines $) \times(7.6$ words/line $) \times(\#$ columns $)$.

- Figures: total 1818.18 words

$=($ figure height in $\mathrm{mm}+10 \mathrm{~mm}) \times(2.2$ words $/ \mathrm{mm}) \times(\#$ columns $)+(\#$ words in caption $)$

Figure 1: $(56.1 \mathrm{~mm}+10 \mathrm{~mm}) \times(2.2$ words $/ \mathrm{mm}) \times 2+7$ words $=297.84$ words

Figure 2: $(50.1 \mathrm{~mm}+10 \mathrm{~mm}) \times(2.2$ words $/ \mathrm{mm}) \times 1+8$ words $=140.22$ words

Figure 3: $(50.1 \mathrm{~mm}+10 \mathrm{~mm}) \times(2.2$ words $/ \mathrm{mm}) \times 2+14$ words $=278.44$ words

Figure 4: $(44.1 \mathrm{~mm}+10 \mathrm{~mm}) \times(2.2$ words $/ \mathrm{mm}) \times 2+25$ words $=263.04$ words

Figure 5: $(114 \mathrm{~mm}+10 \mathrm{~mm}) \times(2.2$ words $/ \mathrm{mm}) \times 1+20$ words $=292.8$ words

Figure 6: $(50.6 \mathrm{~mm}+10 \mathrm{~mm}) \times(2.2$ words $/ \mathrm{mm}) \times 2+44$ words $=310.64$ words

Figure 7: $(35.5 \mathrm{~mm}+10 \mathrm{~mm}) \times(2.2$ words $/ \mathrm{mm}) \times 2+35$ words $=235.2$ words

\section{Supplemental materials: 1}

Color figures in electronic version only. 


\title{
Evolution of $\mathrm{MoO}_{3}$ nanobelts and nanoplatelets formation with flame synthesis
}

Chao Ma ${ }^{\text {a }}$ Xinyao Zou ${ }^{\text {a }}$, Ang Li ${ }^{\text {a }}$, Haozhen Li ${ }^{\text {a }}$, Stelios Rigopoulos ${ }^{\text {b }}$, Lei Zhu ${ }^{\text {a, }}$, Zhen Huang ${ }^{\text {a }}$

\begin{abstract}
A co-flow premixed flat flame is applied to form $\mathrm{MoO}_{3}$ nanobelts and nanoplatelets in the gas phase. An experimental study is conducted with a thermophoretic sampling particle diagnostic (TSPD) technique to reconstruct the evolution of nanostructure formation. In order to investigate the growth mechanism of the nanobelts and nanoplatelets, samples were directly taken along the centerline at different positions in the flame to represent the essential morphological variations of material. Based on the sample information collected by TEM, a growth mechanism of nanobelts and nanoplatelets is proposed. The results indicate that nanobelts and nanoplatelets with well-defined structure are successfully synthesized in premixed flame. The precursor temperature has a significant impact on the morphology by affecting the vapor concentration in the flame. For synthetic nanobelts, the intermediate particles tend to grow along a specific direction with small surface energy, and the final morphology is determined by particle attachment. In contrast, the initial growth of the nanoplatelets is mainly characterized by vapor surface deposition/growth, and the formation of the final morphology relies on the coalescence of large particles and small particles.
\end{abstract}

Keywords: Flame synthesis; Thermophoretic sampling; Growth mechanism; Nanobelts; Nanoplatelets 


\section{Introduction}

Flame synthesis has been proven to be a promising method for nanomaterial synthesis such as nanoparticles, nanowires, nanotubes, and other structured materials [1-3]. Compared with other traditional synthesis methods, flame synthesis provides a natural oxidization environment in which it realizes rapid growth rate, one-step processing and catalyst-free reactions. Flame synthesis, as a very flexible method, can not only be used to control the morphology of the material by adjusting parameters such as flame type, equivalence ratio and temperature, but also enable the nanostructure to have outstanding additional characteristics.

Different ways of flame synthesis have been employed, depending on the type of precursor. As a pioneer in nanoparticle synthesis by flame spray pyrolysis, Pratsinis et al. [4] conducted the numerical simulation and experimental research on the precursors contained in either a vapor or an aerosol. In situ study was conducted to investigate the dynamics of nanoparticle growth in flames volume, focusing on the growth mechanism of nanostructures in flame from the perspective of time scale $[5,6]$. In recent years, more attention has been paid to flame synthesis of metal oxide nanostructures with solid metals as precursors which differs markedly from the aerosol and flame spray pyrolysis method. Flame synthesis with solid precursor is effective method to synthesize multi-dimensional nanostructures and multiple chemical structures in flame volume, and the lower synthesis temperature contribute to maintaining low concentration of precursor to prevent homogeneous nucleation in the gas phase. For example, Merchan-Merchan et al. [7, 8] investigated the formation of carbon and metal oxide nanostructures synthesized on the Mo probe by a counter-flow flame. It was concluded that the flame position (temperature) and probe diameter have great influence on micromorphology. Zheng et al. [2,9] prepared one-dimensional (1D) metal oxide nanostructures by flame vapor deposition. In this experiment, metal wire or mesh was used as precursor, and these nanostructures were applied to photoelectrochemical water splitting. Based on previous research, Kim et al. [10] has developed a flame synthesis system equipped with a wire feeder to 
provide precursors and prepared tungsten oxide nanostructures of different morphologies.

Most researches focused on the property analysis of nanostructure from flame synthesis. As morphology modification is one of the most effective methods to improve the properties of metal oxide nanostructures, it is necessary to investigate the influence of flame parameters on morphology. Those parameters are of great significance for controlling the morphology of nanostructures synthesized by flame. Merchan-Merchan et al. [11-13] conducted an experimental study on the evolution of Mo oxide nanocrystals, carbon-coated $\mathrm{WO}_{3}$ nanoplatelets and nanorods, and mixed tungsten-molybdenum oxide nanostructures in counter-flow flame. Via sampling particles from the flame, it was found that both flame temperature and chemical composition have an effect on the nanostructures. Srivastava et al. [14] employed a counter-flow flame to investigate 1-D Mo oxide nanostructures by experimental and numerical methods. The counter-flow flame involves strong temperature and chemical composition gradients, thus making it impossible to reveal the influence of a single factor on the morphology of nanostructures. In addition, this method, due to its limited flame space, confines the complete growth of nanostructures and is not conducive to the deposition of nanomaterials on the substrate.

In this paper, a co-flow premixed flat flame was applied to construct a synthesis environment with temperature as a single variable. Two different morphologies, $\mathrm{MoO}_{3}$ nanobelts and nanoplatelets with controllable characteristics, could be obtained by controlling the precursor temperature. The thermophoretic sampling particle diagnostic (TSPD) method was used to obtain samples from the flame. Transmission electron microscopy (TEM) was used to investigate the particles' morphology, and the evolution of $\mathrm{MoO}_{3}$ nanobelts and nanoplatelets formation was reconstructed. In addition, a growth mechanism is proposed for the two morphologies. The overall aim of the study is to analyze the formation process of micro morphology and obtain guidelines for determining the flame parameters in order to achieve controllable morphology of nanostructures. 


\section{Experimental methods}

\subsection{Experimental setup}

Figure 1 shows the experimental apparatus in the flame synthesis. A co-flow premixed flat flame is formed from $\mathrm{CH}_{4}$ and air in a McKenna burner with a diameter of $60 \mathrm{~mm}$. The flowrates of the fuel and oxidant are 2 SLPM and 23.8 SLPM, respectively, and they are controlled by a mass flowmeter with an accuracy of $1.0 \%$. A water-cooled cooling plate in the post-flame region is used to improve the flame stability and provide a temperature boundary. The temperature of cooling water was maintained at $20{ }^{\circ} \mathrm{C}$ by the circulating cooling water temperature control system while the substrate's temperature is controlled by adjusting the distance between the substrate and the cooling plate with steel cooling shims. The temperature of precursor is determined by the height position of the precursor above the burner and the number of cooling mesh which inserted below the source mesh. In the $\mathrm{MoO}_{3}$ nanoplatelets synthesis experiment, the cooling plate is also employed as substrate holder. A fluorinated tin oxide coated glass (FTO) substrates are held either by two bolts screwed into the cooling plate in the case of synthetic nanoplatelets or by a tweezer in the case of synthetic nanobelts. A precursor mesh (53 mm length, $32 \mathrm{~mm}$ width, $6 \mathrm{~mm}$ wire spacing, $0.35 \mathrm{~mm}$ diameter and 99\% purity) held in flame volume by ceramic tweezers was used as a source of $\mathrm{Mo}$ for the synthesis of $\mathrm{MoO}_{3}$ nanostructures with various morphologies. All components are mounted on the three-dimensional coordinate frame to enable sampling at various axial locations along the burner centerline.

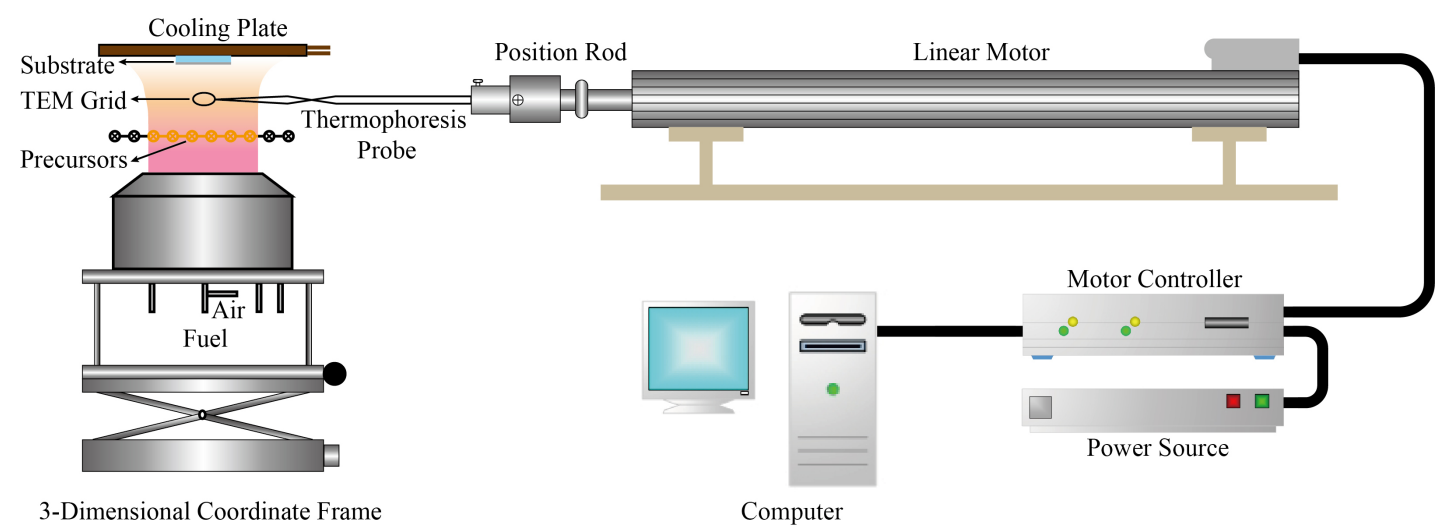


Fig. 1. Schematic of the experimental setup.

The thermophoretic sampling particle diagnostic (TSPD) method is employed to reconstruct the evolution of nanostructure formation [11, 12]. A thermophoretic probe sampler assembled on the position rod is driven in and out of the flame by a linear motor of an electric cylinder (DNCE-32-320-LAS-H, FESTO) controlled by a motor controller (SFC-LACI-VD-10-E-H2-I0, FESTO). A TEM grid is utilized to capture samples from various heights on the flame axis for subsequent TEM characterization. The thermophoresis generated by the temperature gradient between the cold TEM grid and the local flame volume drives the sample deposition. The sampling results might be affected by the residence time of the probe in the hot flame gas, which is limited by a threshold, a critical parameter. Insufficient residence time of the probe in the hot flame might lead to insufficient samples being captured. On the other hand, if the residence time is too long, the surface temperature of the probe cannot prevent reactions between the samples taken and the burning on the probe surface at high temperature. Furthermore, the lower temperature of probe surface can freeze and quench chemical reactions within the samples, which prevents further changes of the morphology and structure of the samples. Based on the results of a preliminary experiment, $50 \mathrm{~ms}$ is the optimal residence time of sampling grid in the flame volume. The trajectory time of probe in and out of the flame is about $30 \mathrm{~ms}$.

\subsection{Sampling and characterization}

In this paper, the sampling positions are at different heights of the flame centerline between the substrate and the metal source precursor. In order to study the evolution of nanostructure formation in the intermediate process by transmission electron microscope, a copper mesh with about $3 \mathrm{~mm}$ diameter supported by ultra-thin carbon film is used to seize samples. $Z$ denotes the distance from the metal source to the sampling position. Prior to sampling, the precursor mesh and substrate are placed at a certain height above the burner, $H$. For the synthesis of nanoplatelets, the Mo mesh and FTO are separately mounted at a distance of $H=30 \mathrm{~mm}$ and $58 \mathrm{~mm}$. For the 
synthesis of nanobelts, the Mo mesh and FTO are mounted at a distance of $H=50 \mathrm{~mm}$ and $78 \mathrm{~mm}$ respectively.

The rapid thermocouple insertion method, similar to the one applied by Gao [15], is employed for temperature measurement. All temperatures involved in the sampling process are measured using uncoated $S$-type thermocouples (Omega Engineering, Inc., $127 \mu \mathrm{m}$ junction size, $50 \mu \mathrm{m}$ wire diameter).

The final morphology of samples on the substrate are investigated by scanning electron microscope (SEM, TESCAN-MAIA3). The synthesized microstructures of samples obtained from different heights of the flame are analyzed by transmission electron microscope (TEM) to understand the evolution process of nanostructure formation in the flame volume between metal source and substrate. SEM and TEM images are visualized and effective method to reflect and analyze the growth process of nanoparticles' morphology. X-ray diffraction (XRD, D8 ADVANCE Da Vinci) measurements are preformed to analyze the crystal structures and phases of the samples. An energy dispersive spectrometer (EDS) analyzer is used to analyze the components and contents of elements of materials.

\section{Results and discussion}

\subsection{Morphology and phase}

Figure 2 shows the temperature profiles from the precursor to the substrate, while the SEM images of representative $\mathrm{MoO}_{3}$ nanobelts and nanoplatelets are shown in the Fig. 3(a) and 3(b). The $\mathrm{MoO}_{3}$ nanobelts grow on substrate for 15 min with $T_{\text {source }}=706.1{ }^{\circ} \mathrm{C}, T_{\text {substrate }}=538.1{ }^{\circ} \mathrm{C}$. The $\mathrm{MoO}_{3}$ nanoplatelets are synthesized at $T_{\text {source }}=866.6^{\circ} \mathrm{C}, T_{\text {substrate }}=498.3{ }^{\circ} \mathrm{C}$ for 15 min with air shielding gas flow rate at $15 \mathrm{~L} / \mathrm{min}$. 


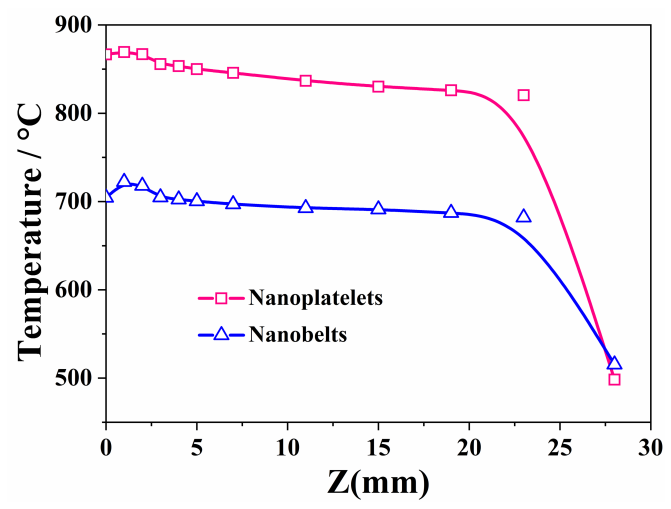

Fig.2. Temperature distribution between the precursor and substrate.

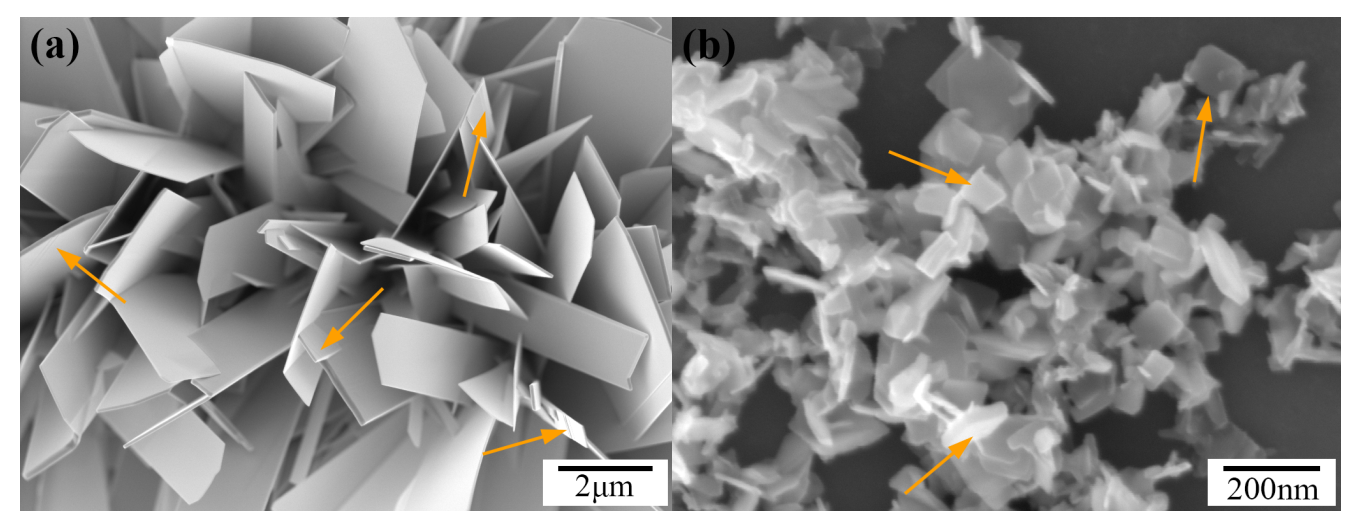

Fig. 3. Top view of SEM images of (a) $\mathrm{MoO}_{3}$ nanobelts and (b) $\mathrm{MoO}_{3}$ nanoplatelets.

As the SEM results shown in Fig. 3, the $\mathrm{MoO}_{3}$ nanobelts with large scale and large coverage density are evenly aligned over the entire substrate, and the morphology of nanobelts features a rectangular ribbon structure with a random direction. The average width and thickness of nanobelts are $79 \mathrm{~nm}$ and $1.3 \mu \mathrm{m}$ respectively. As highlighted by the yellow arrows in Fig. 3(a), layered shapes of $\mathrm{MoO}_{3}$ nanobelts are observed, which indicates a growth of the lateral surface. In the flame synthesis process, the lateral surface is smooth and low in bond energy. Some molecules adhere to the lateral surface and nucleate to form clusters by surface nucleation, which eventually forms a new layer [14]. This results in an increase in the width and thickness of the nanobelts. Fig. 3(b) represents the SEM picture of hybrid $\mathrm{MoO}_{3}$ nanoplatelets, which exhibits large agglomeration on the surface of the substrate. The morphology of most nanoplatelets consists of a cubic structure of small scales, as indicated by the arrows in the Fig. 3(b). In contrast to these two samples, the coverage density of the nanoplatelets is much higher than that of the nanobelts. Some cuboidal nanoplatelets form irregular chain-like shapes of short length 
due to particle fusion. Comparing Fig. 3(a) with Fig. 3(b), we can see that certain parameters in the flame volume exert crucial influence on the morphology. The temperature of metal source for $\mathrm{MoO}_{3}$ nanobelts is lower than that for $\mathrm{MoO}_{3}$ nanoplatelets (as shown in Fig. 2), resulting in low supersaturation ratios of precursors.

The supersaturation ratios is indicated by partial pressure in the gas phase [13]. According to the expression of the indefinite integral of Clausius-Clapeyron, $\ln p=-\frac{\Delta H}{R T}+C$, the partial pressure of the vapor is proportional to the $T_{\text {source. }}$ The $T_{\text {source }}$ for $\mathrm{MoO}_{3}$ nanobelts is lower than that for nanoplatelets, resulting in supersaturation ratios of precursors low enough to prevent homogeneous nucleation, which is ideal for longitudinal growth. For the nanoplatelets, large driving force generated by high supersaturation and low $T_{\text {substrate }}$ is large enough to overcome the surface energy cost, making a uniform growth. For the nanobelts, the small driving force make the growth process occur in the place with small surface energy, limited by surface energy, which contributes to vertical and lateral growth.
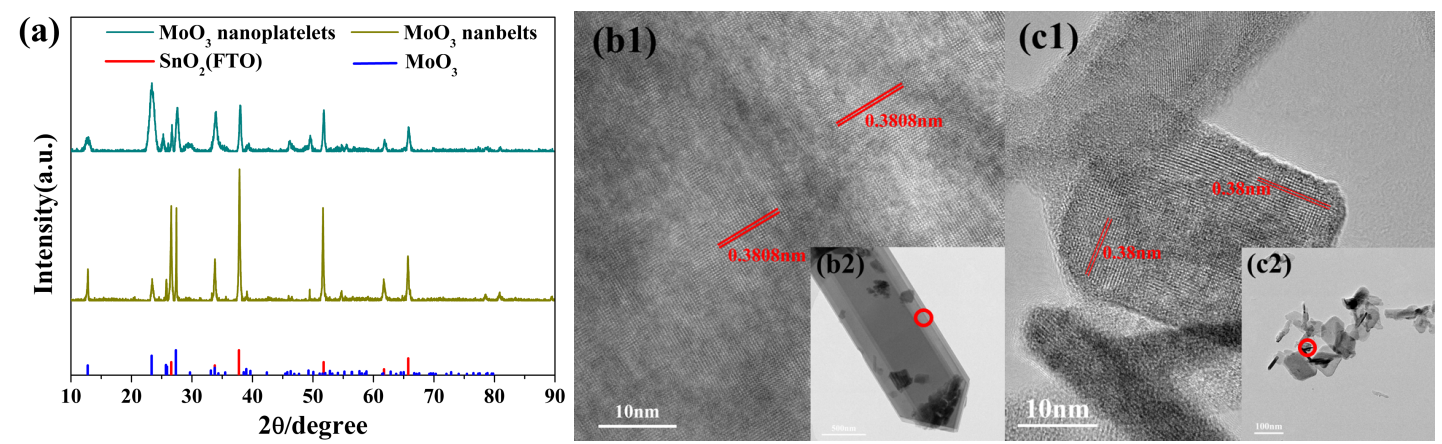

Fig. 4. (a) XRD patterns for pure $\mathrm{MoO}_{3}, \mathrm{SnO}_{2}$, as-grown $\mathrm{MoO}_{3}$ nanobelts, and $\mathrm{MoO}_{3}$ nanoplatelets respectively.

TEM image of: (b) $\mathrm{MoO}_{3}$ nanobelts, (c) $\mathrm{MoO}_{3}$ nanoplatelets. 
Figure 4 shows the details of the morphology of the crystal lattice. The phase and crystalline structures are investigated by XRD, and XRD patterns of as-grown samples are exhibited in Fig. 4(a). The characteristic peaks of $\mathrm{SnO}_{2}$ resulting from growing substrates can be observed in both as-grown $\mathrm{MoO}_{3}$ nanobelts and $\mathrm{MoO}_{3}$ nanoplatelets. There is no shift in the XRD spectra position of nanobelts and nanoplatelets. The $\mathrm{MoO}_{3}$ nanobelts and nanoplatelets exhibit almost the same XRD spectra of standard orthorhombic $\alpha-\mathrm{MoO}_{3}(\mathrm{PDF} \# 35-0609)$ with a distinct peak located at $2 \theta=12.78^{\circ}, 23.339^{\circ}, 27.339^{\circ}$ corresponding to (020), (110), (021) crystal planes respectively. The XRD spectra of the $\mathrm{MoO}_{3}$ nanobelts are sharp and strong in comparison with those of the nanoplatelets, which confirms that the crystallinity of nanobelts is higher than that of nanoplatelets. Fig. 4(b2) is the TEM image of $\mathrm{MoO}_{3}$ nanobelts with rectangular ribbon structures deposited on the substrate. Layered shapes of $\mathrm{MoO}_{3}$ nanobelts could be identified in Fig. 4(b2), which are located at the edge of the nanobelt. The mature sample shows the characteristic spacing of $0.3808 \mathrm{~nm}$ (Fig. 4(b1)) for the (110) lattice planes of $\alpha-\mathrm{MoO}_{3}$ corresponding to $2 \theta=23.339^{\circ}$ in Fig. 4(a). The sample in Fig. 4(c) was $\mathrm{MoO}_{3}$ nanoplatelets obtained from the substrate. The morphology of the hybrid particles is completely different from that of the nanobelts. It is noteworthy that the typical morphology of the sample is cubic particles. High-resolution TEM images in Fig. 4(c1) indicate that the samples present the lattice with an (110) interlayer spacing of $0.38 \mathrm{~nm}$. The EDS analysis is shown in Fig. S1 in supplemental material. 


\subsection{Growth mechanism}

The flame synthesis of Mo nanomaterials is implemented by inserting a precursor in the form of a metal mesh in the flame, as shown in Fig. 1. Samples are captured directly between the precursor and the substrate to reconstruct the evolution of the nanostructures. Prior to sampling, the burner position is adjusted to ensure that the centerline of the TEM grid mounted on the probe coincides with that of the burner during sampling. Fig. 5 shows a series of TEM pictures of acquired microscopic samples at various heights of the centerline of the premixed methane flame.

Based on the results of Fig. 5, a growth mechanism of nanobelts is proposed in this paper. It is assumed that the growth mechanism is based on the following processes: (i) monodisperse particles are produced in flame, (ii) monomers have a tendency to deposit directionally around a cluster, (iii) nucleation and growth of short rod-like particles takes place, (iv) particle attachment promotes vertical and lateral growth of elongated structures. The TEM images in the left column of Fig .5 constitute a visual representation of nanobelt evolution and formation within the flame volume. 


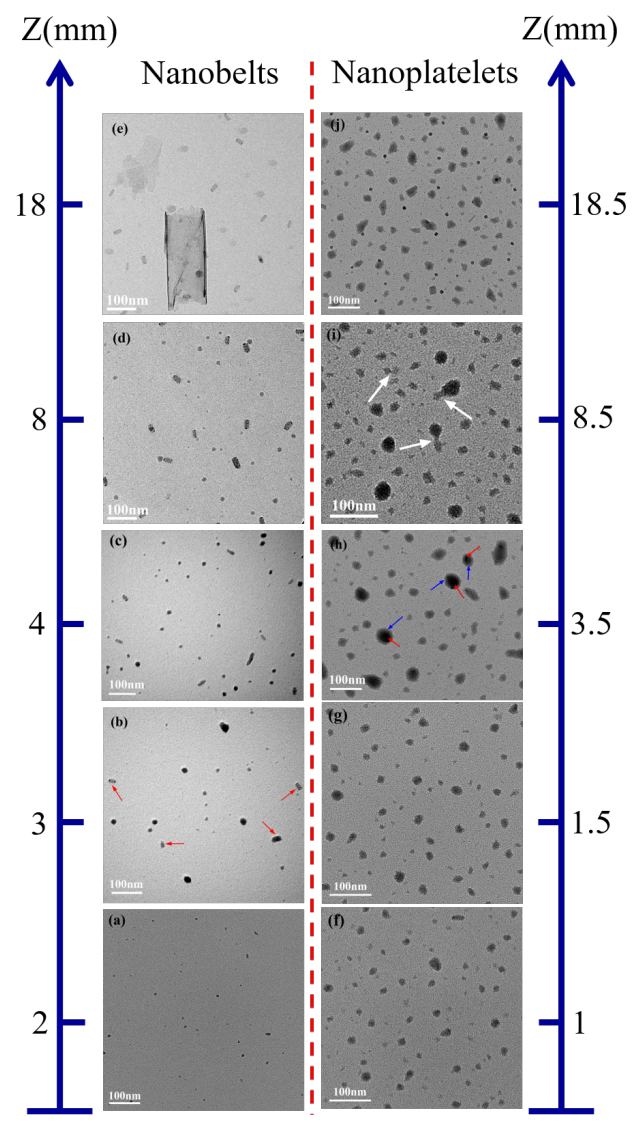

Fig. 5. Representative TEM images of the evolution of nanobelts and nanoplatelets captured at various heights of the flame centerline.

As shown in Fig. 5(a), the sample is collected at the early formation stage at the height of $Z=2 \mathrm{~mm}$. These microstructures, captured by the TEM grid, are randomly distributed with low density. The precursor produced at this stage consists of discrete shapeless small particles with average size $7.76 \mathrm{~nm}$. It can be inferred that the Mo mesh is heated to oxidize and generate vapor at the early stage of flame synthesis, providing a metal source for the growth of the nanomaterial. These amorphous particles near the surface of the Mo mesh are transferred by the combination of thermophoresis and flow field to be converted into intermediate nanostructures, during which is accompanied by some particle collisions and reactions. The XPS analysis is shown in Fig. S2(b) in supplemental material. In Fig. 5(b), the typical morphology of the sample captured at the height of $Z=3 \mathrm{~mm}$ is observed (as shown by the red arrows). It is evident that the sample exhibits an elongated morphology in the form of a short rod-like shape with a rectangular projection from the top view of the electron beam. The average length and width 
of the elongated structures are $23.6 \mathrm{~nm}$ and $11.7 \mathrm{~nm}$ respectively. The nanostructures depicted in Fig. 5(c) have either well defined edges or regular shapes. In comparison to Fig. 5(b), the greater number of structures with short rod-like shapes is shown in Fig. 5(c) with the same magnification. The size of the representative structure collected at the height of $Z=4 \mathrm{~mm}$ has increased to one with a length of $28.63 \mathrm{~nm}$ and a width of $12.07 \mathrm{~nm}$. The precursor tends to orientated growth under the experimental conditions of synthesizing nanobelts, which contributes to the formation of one-dimensional nanostructures.

Short rod-like structures of a larger size are observed in Fig. 5(d). The TEM analysis of the structure sampled at $Z=8 \mathrm{~mm}$ reveals that most samples will be converted into regular shapes with directional growth at that flame position. The representative structures are randomly distributed with an average length of $33.51 \mathrm{~nm}$ and an average width of about $16.89 \mathrm{~nm}$. It can be observed from Fig. 5(e) that the sample obtained farther away from the metal source at the flame position $Z=18 \mathrm{~mm}$ has a mature one-dimensional structure with well-defined elongated shape, showing the morphology of the nanobelts.

The formation processes of $\mathrm{MoO}_{3}$ nanoplatelets are quite different from those of nanobelts, and the differences are mainly due to the different supersaturation ratios of the metal vapor in the flame at different metal source temperatures (as show in Fig. 2). This paper proposes the growth mechanism of $\mathrm{MoO}_{3}$ nanoplatelets as including the following processes: (i) metal oxide vapor is formed, (ii) vapor forms condensed agglomerates in a relatively low temperature region, (iii) surface deposition and growth takes place, nucleus formation, (iv) large particles absorb small particles and gradually grow up to the final size and morphology.

It is observed from Fig. 5(f) that the sample collected at $Z=1 \mathrm{~mm}$ exhibits dispersed monomers with an average size of $16 \mathrm{~nm}$. The diameter and concentration of young particles is greater than those of nanobelts in Fig. 5(a). The XPS analysis is shown in Fig. S2(a) in supplemental material. Samples transported to the height of $Z$ $=1.5 \mathrm{~mm}$ are condensed agglomerates with irregular shapes as shown in Fig. $5(\mathrm{~g})$. The average diameter of 
primary particles is approximately $17.6 \mathrm{~nm}$. The increase in diameter results from the further condensation of the vapor and the formation of amorphous agglomerates conforms to a gas-solid mechanism in flame $[13,16]$.

Primary particles flow further away from the precursor in a relatively low temperature position of $Z=3.5$ $\mathrm{mm}$ and get converted to nascent particles, and the growth mechanism changes from vapor condensation to direct vapor deposition on the surface of the particle [17] as shown in Fig. 5(h). It is hypothesized that the darker and lighter contrast areas pointed out by the red and blue arrows are generated by vapor condensation and direct vapor deposition on the surface. The deposition enables rapid growth of the nascent particles.

The obvious particle merging phenomena shown in Fig. 5(i) indicate that the growth of large particles has been accomplished by absorbing small particles. Such a particle merging behavior reflects randomness and allows the samples to form hybrid nanoplatelets. From Fig. 5(j), it is clearly observed that most of the particles are flake polygons in shape. Compared with the growth process of nanobelts, mature directional growth structure is not captured in the flame environment of particle synthesis. At the height of $Z=18.5 \mathrm{~mm}$, the average diameter of the particles is up to $23.5 \mathrm{~nm}$, and the frequency of occurrence of large particles is greatly increased.

Based on the above results, it is quite obvious that the growth mechanisms of the two structures are different mainly due to the particles' different growth directions. Fig. 6 indicates different growth directions of nascent particles for nanobelts and nanoplatelets.

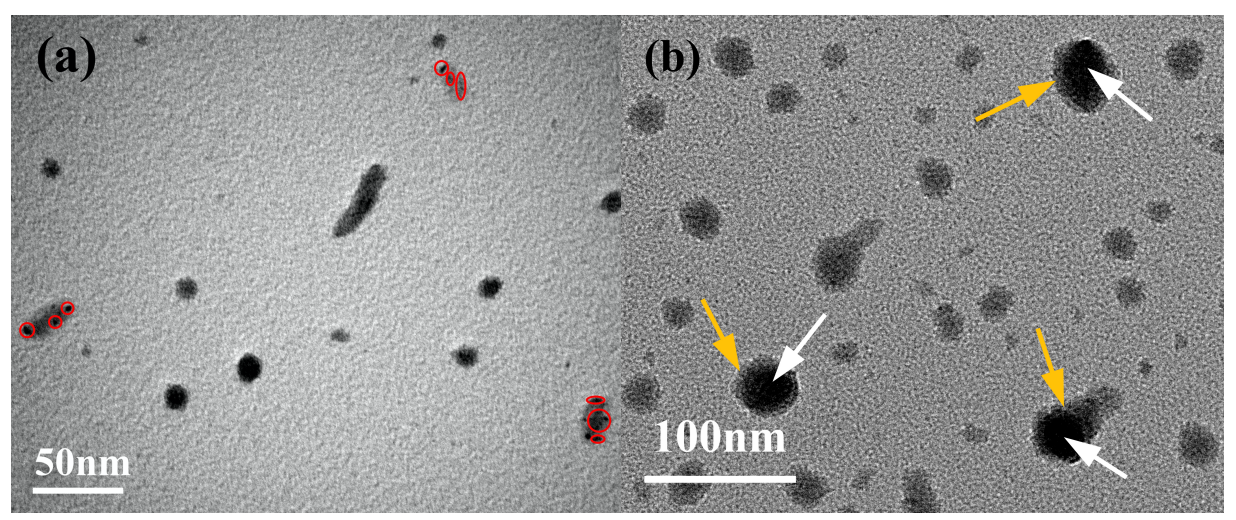

Fig. 6. (a) Sample collected at $Z=4 \mathrm{~mm}$ for nanobelts, red circles indicate small particles that are linearly 
distributed. (b) Sample collected at $Z=5.5 \mathrm{~mm}$ for nanoplatelets; young particles and surface depositions are highlighted by white and yellow arrows respectively.

In Fig. 6(a), the TEM image indicates that these elongated structures are built of small amorphous particles of varying amounts as shown by red circles. The linear arrangement of those particles along a specific direction reveals the directional growth of them. In the case of nanoplatelets, as highlighted by white and yellow arrows in Fig. 6(b), the contrast of dark and light areas indicates that the sample collected at this flame position is composed of two parts. If we can regard the dark region as being formed at an early stage in a position lower than the sampling position, and the light region as being due to further deposition and growth of vapor on the surface during the transport process of young particles, then we can regard the formation of the nanoplatelets as the consequence of the overall rapid growth of the particles, based on the size analysis in Fig. 5.

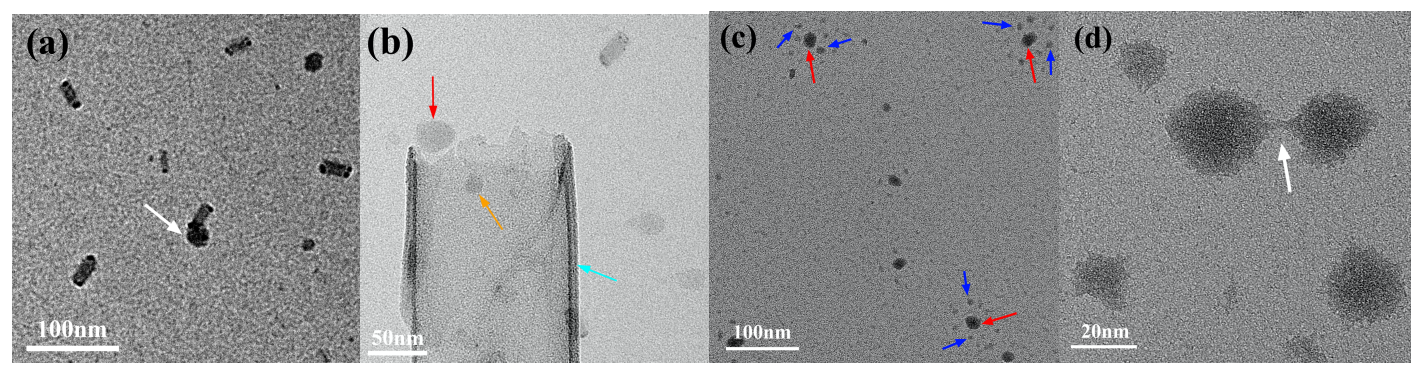

Fig. 7. (a) and (b) the growth patterns of nanobelts collected at $Z=8 \mathrm{~mm}, Z=18 \mathrm{~mm}$, (b) and (c) the growth patterns of nanoplatelets collected at $Z=3.5 \mathrm{~mm}, Z=8.5 \mathrm{~mm}$, respectively.

Furthermore, the formation of typical morphology is related not only to the growth direction but also to the growth pattern of the intermediate particles. The TEM image in Fig. 7(a) shows that the ends of elongated structures bind to the precursor particles. Such a growth pattern promotes the directional increase of size of intermediate particles, causing the vertical growth to be more advantageous than the lateral growth. Fig. 7(b) reveals that the growth of nanobelts in flame medium includes vertical growth and lateral growth. The young particles formed in early formation regions adhere to the rough tip (red arrows), resulting in an increase in length. 
It can be concluded that particles are more likely to be attached onto the tip of elongated structures. In addition, some particles aggregated on the profile (yellow arrow) of mature structure contribute to either width or thickness growth. As pointed out by the blue arrow, the structural characteristic of $\mathrm{MoO}_{3}$ is the layered structure $[3,18]$. In terms of the growth of nanoplatelets, the formation of the final morphology mainly depends on the absorption of small particles by large particles. The large particles are surrounded by several amorphous small particles in Fig. 7(c) collected at $Z=3.5 \mathrm{~mm}$. It is tempting to speculate that large particles will absorb small particles for further growth. As shown in Fig. 7(d), the coalescence of large particles and small particles promotes the growth of amorphous particles to reach the nucleation size and gradually form the final morphology. Such growth patterns exhibit an increase in the size of the intermediate particles rather than in directional growth.

\section{Conclusions}

In this work, the standard orthorhombic $\alpha-\mathrm{MoO}_{3}$ with distinct well-structured nanobelts and nanoplatelets was formed by flame synthesis. The morphology of nanostructure was controlled by adjusting the temperature of precursor. It was found that the variation of precursor temperature affects the supersaturation of evaporated metal oxides vapor in the flame, which has a very significant impact on the growth pattern of intermediate particles.

The evolution of formation of the two morphologies is reconstructed by TSPD technique and TEM. The growth mechanisms of morphologies along the centerline are investigated and compared in the co-flow premixed flat flame. In the case of $\mathrm{MoO}_{3}$ nanobelts, the vertical growth and lateral growth are mainly attributed to particle attachment. Directional growth of intermediate particles for $\mathrm{MoO}_{3}$ nanobelts promotes the formation of nanobelts structure. Additionally, the coalescence of large particles and small particles is the main growth pattern for nanoplatelets. Vapor forms particles by surface deposition, and then the large particles absorb small particles to form hybrid nanoplatelets. 


\section{Acknowledgements}

The authors would like to thank the National Science Foundation of China (Project No. 51436005;

91741120; 51676125) for support. 


\section{References}

[1] Y. Ren, J. Wei, S. Li, In-situ laser diagnostic of nanoparticle formation and transport behavior in flame aerosol deposition, Proc. Combust. Inst. 37 (1) (2019) 935-942.

[2] P.M. Rao, I.S. Cho, X. Zheng, Flame synthesis of $\mathrm{WO}_{3}$ nanotubes and nanowires for efficient photoelectrochemical water-splitting, Proc. Combust. Inst. 34 (2) (2013) 2187-2195.

[3] L. Cai, P.M. Rao, X. Zheng, Morphology-controlled flame synthesis of single, branched, and flower-like $\alpha-\mathrm{MoO}_{3}$ nanobelt arrays, Nano Lett. 11 (2) (2011) 872-877.

[4] M. C. Heine, S. E. Pratsinis, Droplet and particle dynamics during flame spray synthesis of nanoparticles, Ind. Eng. Chem. Res. 44 (16) (2005) 6222-6232.

[5] G. Beaucage, H. K. Kammler, R. Mueller, R. Strobel, N. Agashe, S. E. Pratsinis, T. Narayanan, Probing the dynamics of nanoparticle growth in a flame using synchrotron radiation, Nat. Mater. 3 (6) (2004) 370-374.

[6] A. J. Gröhn, S. E. Pratsinis, A. Sánchez-Ferrer, R. Mezzenga, K. Wegner, Scale-up of Nanoparticle Synthesis by Flame Spray Pyrolysis: The High-Temperature Particle Residence Time, Ind. Eng. Chem. Res. 53 (2014) $10734-10742$.

[7] W. Merchan-Merchan, A.V. Saveliev, L.A. Kennedy, Flame synthesis of molybdenum oxide whiskers, Chem. Phys. Lett. 422 (1) (2006) 72-77.

[8] W. Merchan-Merchan, A.V. Saveliev, V. Nguyen, Opposed flow oxy-flame synthesis of carbon and oxide nanostructures on molybdenum probes, Proc. Combust. Inst. 32 (2) (2009) 1879-1886.

[9] X. Zheng, Rapid catalyst-free flame synthesis of dense, aligned alpha- $\mathrm{Fe}_{2} \mathrm{O}_{3}$ nanoflake and $\mathrm{CuO}$ nanoneedle arrays, Nano Lett. 9 (8) (2009) 3001-3006.

[10] J.-R. Ding, K.-S. Kim, Rapid growth of vertically aligned tungsten oxide nanostructures by flame vapor deposition process, Chem. Eng. J. 300 (2016) 47-53. 
[11] W. Merchan-Merchan, A.V. Saveliev, M. Desai, Volumetric flame synthesis of well-defined molybdenum oxide nanocrystals, Nanotechnology. 20 (47) (2009) 475601.

[12] W. Merchan-Merchan, A.V. Saveliev, S.G. Sanmiguel, M.F. Farahani, Flame volume synthesis of carbon-coated $\mathrm{WO}_{3}$ nanoplatelets and nanorods, J. Nanopart. Res. 14 (12) (2012) 1276.

[13] M. Farmahini-Farahani, A.V. Saveliev, W. Merchan-Merchan, Volumetric flame synthesis of mixed tungsten-molybdenum oxide nanostructures, Proc. Combust. Inst. 36 (1) (2017) 1055-1063.

[14] S. Srivastava, M. Desai, W. Merchan-Merchan, A.V. Saveliev, Volumetric flame synthesis of one-dimensional molybdenum oxide nanostructures, Proc. Combust. Inst. 35 (2) (2015) 2307-2314.

[15] Z. Gao, L. Zhu, C. Liu, A. Li, Z. He, C. Zhang, et al., Comparison of soot formation, evolution, and oxidation reactivity of two biodiesel surrogates, Energy Fuels. 31 (8) (2017) 8655-8664.

[16] A. Gurav, T. Kodas, T. Pluym, Y. Xiong, Aerosol processing of materials, Aerosol Sci. Technol. 19 (4) (1993) $411-452$.

[17] M.J. Height, L. Mädler, S.E. Pratsinis, F. Krumeich, Nanorods of ZnO made by flame spray pyrolysis, Chem. Mater. 18 (2) (2006) 572-578.

[18] L.Q. Mai, B. Hu, W. Chen, Y.Y. Qi, C.S. Lao, R.S. Yang, et al., Lithiated $\mathrm{MoO}_{3}$ Nanobelts with greatly improved performance for lithium batteries, Adv. Mater. 19 (21) (2007) 3712-3716. 


\section{Figure captions}

Fig. 1. Schematic of the experimental setup.

Fig.2. Temperature distribution between the precursor and substrate.

Fig.3. Top view of SEM images of (a) $\mathrm{MoO}_{3}$ nanobelts and (b) $\mathrm{MoO}_{3}$ nanoplatelets.

Fig. 4. (a) $\mathrm{XRD}$ patterns for pure $\mathrm{MoO}_{3}, \mathrm{SnO}_{2}$, as-grown $\mathrm{MoO}_{3}$ nanobelts, and $\mathrm{MoO}_{3}$ nanoplatelets respectively.

TEM image of: (b) $\mathrm{MoO}_{3}$ nanobelts, (c) $\mathrm{MoO}_{3}$ nanoplatelets.

Fig. 5. Representative TEM images of the evolution of nanobelts and nanoplatelets captured at various heights of the flame centerline.

Fig. 6. (a) Sample collected at $Z=4 \mathrm{~mm}$ for nanobelts, red circles indicate small particles that are linearly distributed. (b) Sample collected at $Z=5.5 \mathrm{~mm}$ for nanoplatelets; young particles and surface depositions are highlighted by white and yellow arrows respectively.

Fig. 7. (a) and (b) the growth patterns of nanobelts collected at $Z=8 \mathrm{~mm}, Z=18 \mathrm{~mm}$, (b) and (c) the growth patterns of nanoplatelets collected at $Z=3.5 \mathrm{~mm}, Z=8.5 \mathrm{~mm}$, respectively. 


\section{Supplemental materials list}

\section{SMM.doc}

Fig. S1. (a) EDS of elemental spectrum of $\mathrm{MoO}_{3}$ nanoplatelets obtained using TEM. (b) EDS of elemental spectrum of $\mathrm{MoO}_{3}$ nanobelts obtained using TEM.

Fig. S2. XPS spectra of Mo 3d obtained from (a) $\mathrm{MoO}_{3}$ nanoplatelets and (b) $\mathrm{MoO}_{3}$ nanobelts. 